corresponding to the transition from one state to the other is calculated to be about $39,000 \mathrm{~cm} .^{-1}$--almost exactly the observed value. When suitable refinements are made in the theory, the agreement with experiment is still almost exact. This part of our work may be regarded as an extension of a theory of the origin of the absorption bands of benzene proposed by Sklar ${ }^{2}$.

Similar calculations on butadiene show that the central C-C link has a length $1.42 \mathrm{~A}$. in the excited state, compared with $1.43 \mathrm{~A}$. in the ground state, and the outer links have lengths $1 \cdot 61 \mathrm{~A}$., compared with $1.34 \mathrm{~A}$. in the ground state. The energy difference between the two states is $45,000 \mathrm{~cm} .^{-1}$, compared with the experimental value $48,000 \mathrm{~cm} \cdot \cdot^{-1}$. For this molecule, it will be noticed, the internuclear distances in the two states are very different, and as a result, the band in the fluorescent spectrum arising from the transition from one to the other should show considerable vibrational structure.

Calculations on more complicated polyenes have also been made, with results similar to those quoted for butadiene.

The theory employs only the double bond and benzene bond energies and force constants. Now the force constants are known within a few per cent, and in any event their importance in the theory is only subsidiary. Thus the only uncertainty is that arising from the unknown value of the heat of sublimation of carbon, placed by various observers between 130 and $170 \mathrm{kcal} . / \mathrm{mol}$. Pauling ${ }^{3}$ adopts the latter value. Support for his view may be obtained as follows. Suppose that the heat of sublimation is changed to $(170-\Delta) \mathrm{kcal} . / \mathrm{mol}$. Then the calculated value of the energy of the absorption band of benzene mentioned above is decreased roughly by $8 \Delta / 7$. Thus if $\Delta=40$, the calculated energy of the band is changed from $111 \mathrm{kcal} . / \mathrm{mol}$. to $65 \mathrm{keal} . / \mathrm{mol}$., the observed value being 110 . Similarly, the agreement between theory and experiment for butadiene is completely spoiled. We conclude that the heat of sublimation of carbon is 170 kcal./mol., with a possible error of $10 \mathrm{kcal} . / \mathrm{mol}$. Full details will be published later.

$$
\begin{aligned}
& \text { G. J. KYNCH. } \\
& \text { E. H. LloYd. } \\
& \text { W. G. PENNEY. }
\end{aligned}
$$

Imperial College of Science and Technology, London, S.W.7. April 15.

1 Trans. Faryd. Soc., 35, 835 (1939).

2.J. Chem. Phys., 5, 669 (1937).

s J. Amer. Chem. Soc., 54, 3570 (1932).

\section{Structure of the OD Bands of Heavy Water}

Bands due to the OD molecule have been photographed with a large quartz Littrow and a $10-\mathrm{ft}$. concave grating spectrograph by using discharges through vapour of heavy water of a high degree ( 99.6 per cent) of purity. The bands at $\lambda 2872$ and $\lambda 2916$ have been measured. They correspond to the $(1,0)$ and $(2,1)$ bands of $\mathrm{OH}$ with heads at $\lambda 2811$ and $\lambda 2875$ due to the electronic transition ${ }^{2} \Sigma+\rightarrow^{2} \pi_{\text {inv }}$. With the aid of the rotational differences derived from the six main $P, Q$ and $R$ branches the mean values of the constants for the band $\lambda 2872$ are found to be (in $\mathrm{cm}^{-1}$ ) as follow:

$$
\begin{array}{lll}
B_{v}^{\prime}=8.736 \quad ; & D_{v}^{\prime}=0.548 \times 10^{-3} \\
B_{v}^{\prime \prime}=9.870 & ; & D_{v}^{\prime \prime}=0.420 \times 10^{-3}
\end{array}
$$

The values $\mathrm{B}_{v}^{\prime}$ and $B^{\prime \prime}$ are in good agreement with those calculated from the corresponding constants of the $\mathrm{OH}$ molecule using the mass ratio, $P^{2}=0.5301$ $\left({ }^{1} \mathrm{H}=1.0081\right.$ and $\left.{ }^{2} \mathrm{H}=2.0142\right)$ for the two isotopic molecules $\mathrm{OH}$ and $\mathrm{OD}$. The wave numbers of $R_{1}(1)$ and $Q_{1}(1)$ are $34796 \cdot 9$ and $34761 \cdot 6 \mathrm{~cm} .^{-1}$ respectively.

Full details of the structure will be published elsewhere.

\section{Andhra University, Waltair. March 14.}

K. R. RAO.

M. G. SASTRY.

\section{The Generalized Kaleidoscope}

Prof. H. S. M. Coxeter, in his recent admirable essay $^{1}$ on polyhedra, has brought the generalized kaleidoscope to the notice of a wider public. In the accounts of this apparatus which I have seen, there is no reference to those cases in which the angle between two of the mirrors is $120^{\circ}$. The trihedral examples, though interesting, are perhaps of less importance than the case described below.

It is said to have been proved that a kaleidoscope cannot have more than six mirrors; it is, however, possible to have one with eight arranged in a manner suggested by the faces of the rhombic dodecahedron. Take four mirrors the shape of congruent isosceles triangles with the angle at the vertex equal to $70^{\circ} 32^{\prime}$, and arrange them so that they form the slant faces of a pyramid on a square base. (The images of a pointobject at the centre of the base, including the object itself, form the vertices of an octahedron). Taking another set of four mirrors, arranged as before, and bringing the two sets together base to base, an octahedron of mirrors is obtained, the dihedral angles of which are $120^{\circ}$ and $90^{\circ}$. The images of a point-object inside it, together with the object itself, form the vertices of a solid tessellation of cuboctahedra and octahedra.

Winkinghurst, Carr Lane,

Acomb, York. April 9.

'Ball, W. W. R., "Math. Recreations and Essays" (1939), chapter v.

\section{Carbohydrate Metabolism of Tumours}

RECEnT correspondence by Drs. Boyland and Dickens in NATURE has raised once again the question of the existence of an abnormal carbohydrate metabolism of tumours.

Dr. Dickens ${ }^{1}$ maintains that, whilst neither aerobic glycolysis alone nor in combination with a low respiratory quotient is "in the strictest sense specific for tumours, . . . nevertheless, the association in tumour metabolism of relatively high aerobic and anaerobic glycolysis with the lowered respiratory quotient is such a constant one that to dismiss it is to discard as unimportant the most characteristic of established biochemical peculiarities of tumour tissue". We agree with Dr. Dickens that this type 